\title{
PECAS: A Low-Cost Prototype for the Estimation of Channel Activity Statistics in Cognitive Radio
}

\author{
Miguel López-Benítez ${ }^{*}$, Ahmed Al-Tahmeesschi*, Kenta Umebayashi ${ }^{\dagger}$ and Janne Lehtomäki ${ }^{\ddagger}$ \\ ${ }^{*}$ Dept. of Electrical Engineering and Electronics, University of Liverpool, United Kingdom \\ ${ }^{\dagger}$ Graduate School of Engineering, Tokyo University of Agriculture and Technology, Japan \\ ${ }^{\ddagger}$ Centre for Wireless Communications, University of Oulu, Finland
}

\begin{abstract}
Dynamic spectrum access/cognitive radio (DSA/CR) systems can benefit from an accurate knowledge of the primary channel ON/OFF activity statistics. This information can be readily obtained from spectrum sensing observations. However, given the practical limitations of spectrum sensing, an accurate and realistic evaluation of estimation methods is required before they can be implemented in real systems. This paper presents the design and implementation of a low-cost Prototype for the Estimation of Channel Activity Statistics (PECAS) in DSA/CR systems. An in-depth description of the hardware design (based on common and inexpensive components) and software implementation (based on free open source code) is provided along with an illustrative applicability example. The developed platform provides researchers and engineers with a low-cost fully functional tool for proof-of-concept, validation and optimisation of algorithms and designs.
\end{abstract}

Index Terms-Dynamic spectrum access, cognitive radio, primary activity statistics, prototyping.

\section{INTRODUCTION}

Dynamic Spectrum Access/Cognitive Radio (DSA/CR) [1, 2] aims at increasing the efficiency of spectrum use by allowing unlicensed (secondary) users to opportunistically access licensed spectrum bands temporarily unused by the licensed (primary) users. The performance of DSA/CR systems highly depends on the spectrum occupancy pattern of primary users. Therefore, DSA/CR systems can benefit from an accurate knowledge of primary spectrum occupancy statistics. This information can be exploited to predict future spectrum occupancy trends [3, 4], select the most appropriate band/channel of operation [5-8], or make appropriate spectrum and radio resource management decisions [9-13] in order to improve the DSA/CR system performance, protect primary users from interference, and increase the overall spectrum efficiency.

DSA/CR systems can employ various techniques to obtain statistical information on the primary spectrum activity [2]. A simple method is to exploit spectrum sensing observations to this end [14]. The idle/busy information provided by periodic spectrum sensing decisions can be exploited to estimate the durations of the channel idle/busy periods, which can be further processed to obtain more elaborated statistical information (e.g., minimum/maximum channel idle time, channel duty cycle, statistical moments such as mean and variance, or the underlying distributions for the channel holding times).

Compared to other alternatives such as the use of databases, the sensing-based approach has significant advantages, includ- ing low cost and complexity, independence (the DSA/CR system does not need to rely on external information systems) and suitability to a wide range of spectrum bands, including those with highly dynamic occupancy patterns. However, its main drawback is the limited reliability arising from an imperfect detection performance (i.e., sensing errors) and other practical limitations [14], which can affect severely the accuracy of the estimated channel activity statistics [15].

Some methods have been proposed for the estimation of primary channel activity statistics based on (imperfect) spectrum sensing observations [15-18]. However, existing studies are based either on mathematical analyses or simulations, which lack of sufficient realism and accuracy, or evaluation platforms usually based on rather expensive equipment. A simple yet realistic hardware evaluation platform enabling a wide range of experiments at an affordable cost would be desirable.

In this context, this paper presents the design and implementation of a low-cost Prototype for the Estimation of Channel Activity Statistics (PECAS) in DSA/CR systems. In particular, this paper provides an in-depth description of the hardware and software components, discussing implementation details that may be helpful to other researchers and engineers in the development of similar tools. An example illustrating the potentials and capabilities of the developed platform is presented as well. The presented prototype is based on common components with an approximated total cost of $£ 60 / 70 € / \$ 80$ along with free open source $\operatorname{code}^{1}$. The developed platform provides researchers and engineers with a low-cost fully functional tool for proof of concept, validation and optimisation of algorithms and designs before bringing them to real systems.

The rest of this paper is organised as follows. First, Section II provides a general overview of the developed prototype. A more detailed description of the two main modules of the prototype, namely the transmitter and the receiver, is provided in Sections III and IV, respectively. An illustrative example on the potential applicability of the prototype along with experimental results are presented in Section V. Finally, Section VI summarises and concludes the paper.

\section{OVERVIEW}

The block diagram of PECAS is shown in Fig. 1. The design is composed of two modules: 1) a (primary) transmitter, which

\footnotetext{
${ }^{1}$ Available at: www. lopezbenitez .es/misc/PECAS . zip
} 


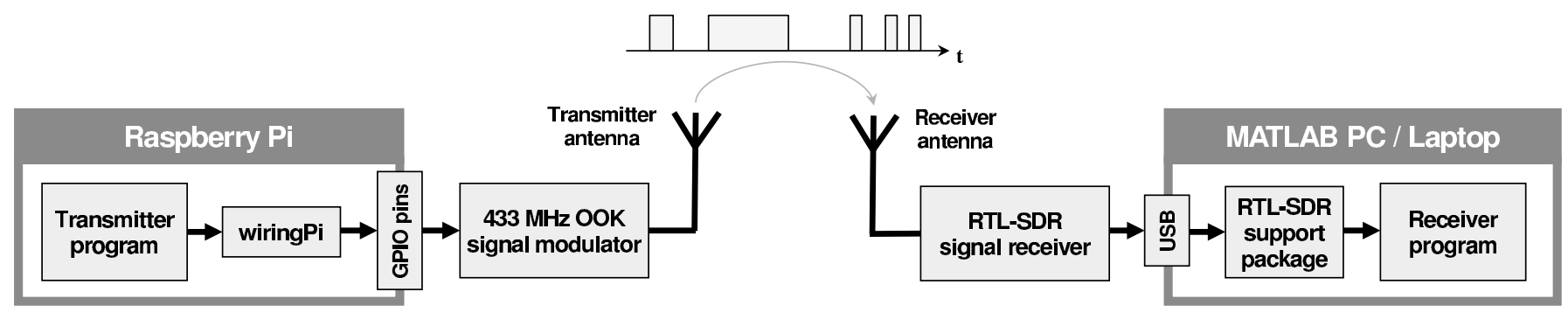

Fig. 1. Block diagram of PECAS.

sends a controlled sequence of ON/OFF periods with known and predefined statistical properties, and 2) a (secondary or DSA/CR) receiver, which senses the channel periodically, estimates the duration of the ON/OFF periods, and processes this information to estimate the statistical properties of the transmitter's activity pattern (e.g., the minimum/maximum idle/busy period durations, their mean and variance, the channel duty cycle, or the underlying distributions of idle/busy periods). By comparing the parameters configured in the transmitter with the estimations obtained in the receiver, it is possible to assess the accuracy of an estimation method or algorithm (such as those in [15-18]) under realistic evaluation conditions, including the channel degrading effects and other practical limitations of real transmitters and receivers.

The prototype operates in the $433 \mathrm{MHz}$ Industrial, Scientific and Medical (ISM) band. The motivation for using this band is manifold. First, experiments in ISM bands are possible without a spectrum license. Moreover, this is a popular ISM band with a wide variety of readily available low-cost radio components, which are typically less expensive than their counterparts designed to operate at higher frequencies in the $2.4 / 5 \mathrm{GHz}$ ISM bands. Moreover, this band is in general less crowded than the $2.4 \mathrm{GHz}$ ISM band. Even though some other transmitters may be present, they are low-power short-range devices, which enables a more easily controllable radio environment free of undesired interference from other potential transmitters.

\section{PECAS TRANSMITTER}

\section{A. Hardware}

The hardware implementation of the PECAS transmitter is shown in Fig. 2. The core component is an ON-OFF Keying (OOK) modulator, which is employed to generate a sequence of ON/OFF periods in the channel. OOK is a binary form of Amplitude-Shift Keying (ASK) that uses the presence of a fixed-amplitude carrier wave to represent a binary one and its absence to represent a binary zero. The transmission (absence) of the carrier wave can be controlled by setting the modulator's data pin to a logical one/high (zero/low) voltage level, which is controlled by a program running in a Raspberry Pi (RBPi).

While DSA/CR systems may employ other more sophisticated modulations (e.g., orthogonal frequency-division multiplexing), the use of $\mathrm{OOK}$ is here sufficient and adequate.

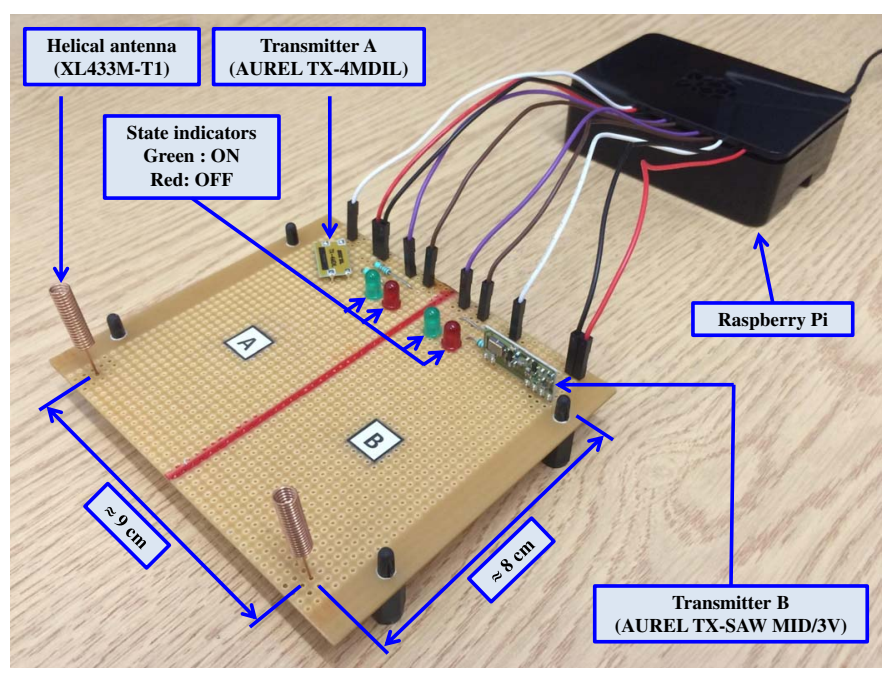

Fig. 2. Hardware implementation of the PECAS transmitter.

First, the signal modulation format is irrelevant when spectrum sensing is based on energy detection [19], which is the most commonly used signal detection method in DSA/CR [20] and the one implemented in the PECAS receiver. Moreover, OOK modulators are extremely easy to control (via a single data pin) and notably less expensive than other modulator circuits (due to the simplicity of the OOK modulation method), which makes of it a suitable candidate for the objectives of this work.

The implementation shown in Fig. 2 features two transmitters based on OOK modulators AUREL TX-4MDIL (A) and AUREL TX-SAW MID/3V (B). Both modulators operate at a central frequency of $433.92 \mathrm{MHz}$ and their voltage and current levels are compatible with the RBPi capabilities (3.3 $\mathrm{V}, 6 \mathrm{~mA})$; the main difference is the output power $(\approx 2 \mathrm{dBm}$ for the former, $\approx 10 \mathrm{dBm}$ for the latter). The presence of both modulators enables a wider range of experiments, including different transmission power levels and aggregated primary transmitters in the same channel. The maximum modulation frequencies supported by the modulators correspond to minimum ON/OFF period durations of $0.25 \mathrm{~ms}(\mathrm{~A})$ and $0.1 \mathrm{~ms}(\mathrm{~B})$; however this is limited to $1 \mathrm{~ms}$ by the software capabilities (see Section III-B). Light Emitting Diodes (LEDs) are used to indicate visually the instantaneous ON (green) or OFF (red) 
state of each transmitter, which can be useful for testing and debugging. All components are powered and controlled from a RBPi through the General-Purpose Input/Output (GPIO) pins, including power supply (red cables), ground (black), ON/OFF modulation data (white), ON state indicator (violet), and OFF state indicator (brown). Finally, the signal is transmitted by $433 \mathrm{MHz}$ helical antennas (XL433M-T1) with $2.2 \mathrm{dBi}$ gain (placed at least $5 \mathrm{~cm}$ apart from other circuits or metal parts).

\section{B. Software}

A program (written in $\mathrm{C}$ ) running in the RBPi controls the OOK modulators through the GPIO pins. This is accomplished with wiringPi, a GPIO interface library for the RBPi that simplifies the access to the GPIO pins from $\mathrm{C} / \mathrm{C}++$ programs $^{2}$.

The duration of the transmitted ON/OFF periods is controlled by setting the modulator's data pin to the desired logical level (one/high for ON periods, zero/low for OFF periods) and calling C's delay function to produce a delay equal to the desired period duration. The input argument for this function is an integer number indicating the desired delay in milliseconds. Therefore, the minimum period duration is limited to $1 \mathrm{~ms}$. Other functions that could be used for shorter delays (such as usleep or nanosleep) are either deprecated or may lead to some interruption problems. The delay function is selected for simplicity and reliability. Moreover, a 1-ms time resolution can be considered sufficiently realistic for most practical experiments and proof-of-concept validations.

The transmitter's control program can be configured to select the desired transmitter (either A or B). The transmitted sequence of ON/OFF periods can be loaded from a plain text file or generated randomly by the transmitter's control program (and, optionally, saved into a file). In both cases, the number of ON/OFF periods to be transmitted can be configured.

Random periods can be generated based on the distributions shown in Table I of [21] (i.e., exponential, generalised exponential, Pareto, generalised Pareto, log-normal, gamma and Weibull), which provide accurate fits to empirical data from real systems [21]. Random periods are generated based on the inversion method [22, p. 28], which exploits the fact that if $F(\cdot)$ is a continuous $\mathrm{CDF}$ on $\mathbb{R}$ with inverse $\mathrm{CDF}$ given by $F^{-1}(\cdot)$, and $U$ is a uniform random variable within the interval $[0,1]$, then the CDF of $F^{-1}(U)$ is $F(\cdot)$. Based on this property, random periods from any distribution $F(\cdot)$ can be generated based on uniform random number generators (such as C's rand function) and the inverse $\mathrm{CDF} F^{-1}(\cdot)$.

Inverting some functions may not be straightforward and may require approximations or numerical methods (e.g., the error and lower incomplete gamma functions in Table I of [21]). To overcome this problem in an efficient and easily extensible manner, inversion is performed numerically following a binary (half-interval) search approach. This approach can be employed to invert monotonically increasing or decreasing functions. Given a function $y=g(x)$, an appropriate interval $\left[x_{\min }, x_{\max }\right]$ is first set, where the target value $x^{*}=g^{-1}\left(y^{*}\right)$

\footnotetext{
${ }^{2}$ Available at: http://wiringpi.com
}

is expected to be (the limits may be readjusted if needed) and the middle point is computed $x_{\text {mid }}=\left(x_{\min }+x_{\max }\right) / 2$. If $g(x)$ is a monotonically increasing function (i.e., $x_{a}<x_{b} \Leftrightarrow$ $\left.g\left(x_{a}\right)<g\left(x_{b}\right)\right)$, the search interval is then iteratively halved as follows: if $y^{*}>g\left(x_{\text {mid }}\right)$ then $x_{\text {min }}=x_{\text {mid }}$, otherwise $x_{\max }=x_{\text {mid }}$. For monotonically decreasing functions (i.e., $\left.x_{a}<x_{b} \Leftrightarrow g\left(x_{a}\right)>g\left(x_{b}\right)\right)$, the search interval is iteratively halved as follows: if $y^{*}<g\left(x_{\text {mid }}\right)$ then $x_{\text {min }}=x_{\text {mid }}$, otherwise $x_{\max }=x_{\text {mid }}$. The middle point is recomputed and the process is repeated until $\left|y^{*}-g\left(x_{\text {mid }}\right)\right|<10^{-10}$. The resulting $x_{\text {mid }}$ is the desired value $x^{*}=g^{-1}\left(y^{*}\right)$. Note that this process can converge to an arbitrarily accurate value and is significantly more efficient than an exhaustive search.

The program also includes a test mode (where a sequence of 1-second ON/OFF periods is transmitted) and can easily be extended to include other new distributions as well.

\section{PECAS RECEIVER}

\section{A. Hardware}

The PECAS receiver is based on RTL-SDR [23]. RTL-SDR is a very low cost Software Defined Radio (SDR) that employs a DVB-T TV tuner dongle based on the Realtek RTL2832U chipset. By means of a suitable driver, the received I/Q data can be accessed directly and processed in software from a PC.

This implementation employs the most common tuner dongle (Raphael Micro R820T, see Fig. 3), which can be used as an SDR receiver in the frequency range 24-1766 MHz (other variants may have different frequency ranges but most of them cover the $433 \mathrm{MHz}$ ISM band). Since they are intended for TV, RTL-SDR dongles are typically provided with a 75-ohm antenna (a 50-ohm antenna could be used with a mismatch loss of $0.177 \mathrm{~dB}$ ). The receiver tolerates a maximum input power of $10 \mathrm{dBm}$ and features a low-noise amplifier with automatic/manual gain control within the range $\approx 0-50 \mathrm{~dB}$.

The signal at $433.92 \mathrm{MHz}$ is down-converted to an intermediate frequency of $3.57 \mathrm{MHz}$ where it is sampled at $28.8 \mathrm{MHz}$ and decimated to the desired base-band sample rate before being sent to the PC via USB. The receiver can provide 8bit I/Q samples at a programmable sample rate (theoretical maximum of $3.2 \mathrm{MS} / \mathrm{s}$, stable maximum of about $2.4 \mathrm{MS} / \mathrm{s}$ ).

\section{B. Software}

A MATLAB program running in a PC / laptop receives and processes the I/Q signal samples from the RTL-SDR receiver by making use of MATLAB's Support Package for RTL-SDR Radio, which provides a convenient programming interface.

The program first configures the RTL-SDR receiver parameters. The central frequency is set equal to the transmitter frequency (i.e., $433.92 \mathrm{MHz}$ ). Other parameters such as the sample rate, automatic/manual tuner gain, frequency correction factor and output data type (integer or single/double precision floating point values) can be configured as desired.

After configuring the RTL-SDR receiver, the program captures and discards 100 frames of signal samples. This is the approximate amount of frames it takes the RTL-SDR receiver to produce reliable data when tuned to a new frequency [23]. 


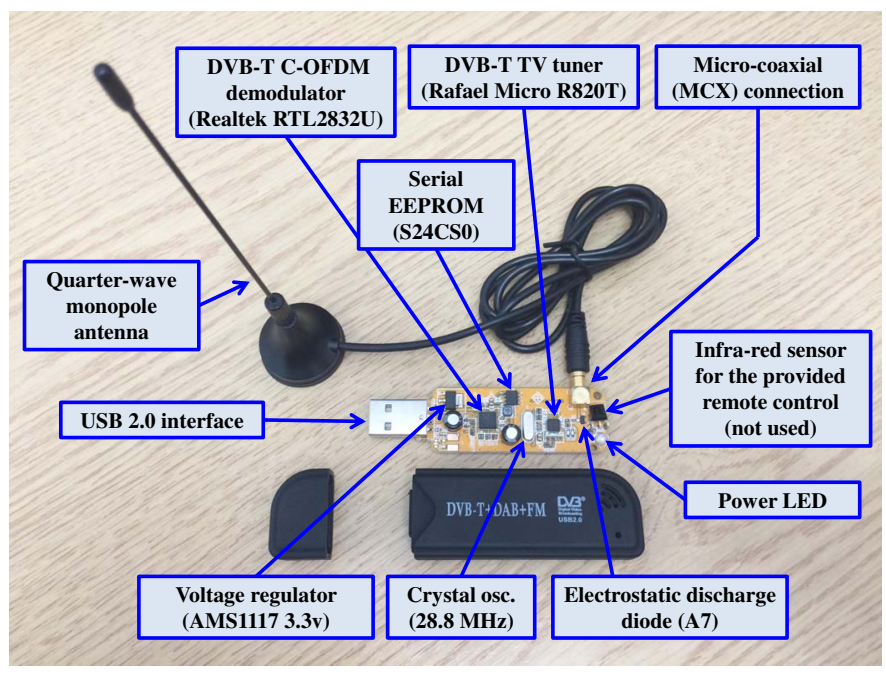

Fig. 3. PECAS receiver (RTL-SDR based on Rafael Micro R820T).

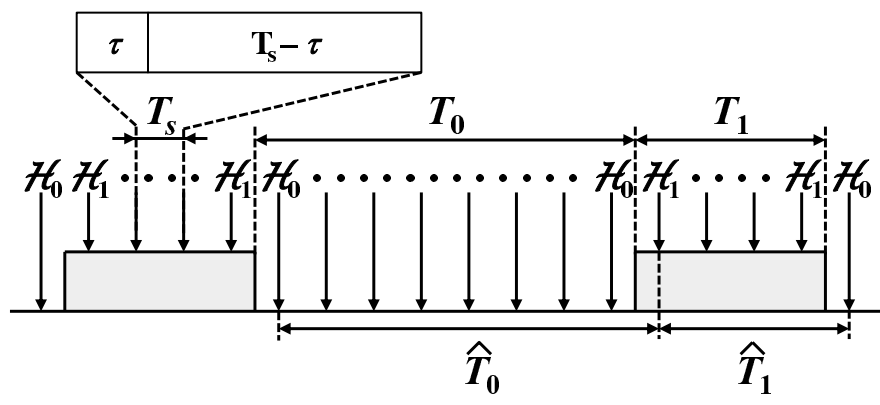

Fig. 4. Estimation of period durations from spectrum sensing decisions.

The program then senses the channel at $433.92 \mathrm{MHz}$ for a pre-configured period of time. During this time, the program estimates the durations of the channel's idle/busy periods following a typical periodic sensing approach as illustrated in Fig. 4. Sensing in DSA/CR systems is typically performed following a periodic sensing frame structure with duration/periodicity $T_{s}$, where each frame consists of one sensing slot with duration $\tau$ (where primary signal samples are captured to make a binary idle/busy decision on the channel state) and a transmission slot with duration $T_{s}-\tau$ (where DSA/CR users may transmit if the channel is decided to be idle). The receiver program emulates this periodic sensing approach by capturing signal samples for intervals of $\tau$ seconds every $T_{s}$ seconds. To this end, a sensing event is scheduled every $T_{s}$ seconds. In every sensing event, the program instructs the RTL-SDR receiver to produce a sufficiently large frame of signal samples. The minimum number of samples required to ensure that the channel is sensed for at least $\tau$ seconds is $N=\left\lceil\tau f_{s}\right\rceil$, where $f_{s}$ is the sample rate configured in the RTL-SDR receiver. However, since the device can only provide samples in blocks that are integer multiples of 256, signal samples are captured in blocks of $\lceil N / 256\rceil \cdot 256$ samples and only the first $N$ samples are considered (the rest of samples are discarded).
In every sensing event, a block $\{x[n]\}_{n=1}^{N}$ of $N$ signal samples is captured and processed according to the energy detection method [19] to produce a binary decision on the channel state. In particular, the channel is assumed to be busy $\left(\mathcal{H}_{1}\right)$ if the energy $\mathcal{E}_{x}$ of the $N$ samples is above a predefined decision threshold $\lambda$, or idle $\left(\mathcal{H}_{0}\right)$ otherwise:

$$
\mathcal{E}_{x}=\sum_{n=1}^{N}|x[n]|^{2} \underset{\mathcal{H}_{0}}{\stackrel{\mathcal{H}_{1}}{\gtrless}} \lambda
$$

In practice, the threshold $\lambda$ is frequently chosen to meet a certain probability of false alarm $P_{f a}$, which only requires the receiver's noise to be known [24]. To select the threshold, the receiver program can be configured to save the observed energy values $\mathcal{E}_{x}$ to a file for post-processing and/or show relevant statistics of $\mathcal{E}_{x}$ (e.g., distribution, percentiles, maximum value, etc.). When the captured samples $x[n]$ are noise-only (i.e., the transmitter remains inactive), the provided statistics can be used to set the energy decision threshold (e.g., the 99th percentile is the value that leads to $P_{f a}=1 \%$ ).

Based on energy detection, the receiver program produces a binary idle/busy $\left(\mathcal{H}_{0} / \mathcal{H}_{1}\right)$ decision every $T_{s}$ seconds. The sequence of spectrum sensing decisions can be exploited to provide estimations $\widehat{T}_{i}(i \in\{0,1\})$ of the real durations $T_{i}$ of the channel's idle/busy periods as shown in Fig. 4. The estimated periods $\widehat{T}_{i}$ are finally saved to a file for postprocessing and analysis. For example, the estimated periods $\widehat{T}_{i}$ can be compared to the actual periods $T_{i}$ generated by the transmitter to determine the impact of the employed sensing period $T_{s}$, sensing duration $\tau$, sample size $N$, probability of sensing errors (e.g., $P_{f a}$ ), etc. The estimated periods can also be processed to estimate the statistical properties of the transmitter's activity pattern (e.g., the minimum/maximum idle/busy period durations, their mean and variance, the channel duty cycle, or the underlying distributions of idle/busy periods). By comparing the parameters configured in the transmitter with the estimations obtained in the receiver, it is possible to assess the accuracy of an estimation method or algorithm (such as those in [15-18]) under realistic evaluation conditions, including the channel degrading effects and other practical limitations of real transmitters and receivers.

\section{Illustrative EXAMPLE AND Results}

In this section an example of applicability of the developed prototype is shown. In this example, the prototype is employed to run an experiment aimed at determining the impact of the sensing period $T_{s}$ and probability of false alarm $P_{f a}$ on the accuracy of the estimated distribution of idle periods.

The transmitter is configured to transmit a previously generated and saved sequence of 300 random ON/OFF periods (600 periods in total) obtained from a generalised Pareto distribution, which was found in [21] to provide the most accurate fit to empirical data from real systems. The distribution is configured with parameters $\mu_{0}=\mu_{1}=100 \mathrm{~ms}$ (location), $\lambda_{0}=\lambda_{1}=300 \mathrm{~ms}$ (scale), and $\alpha_{0}=\alpha_{1}=0.25$ (shape), which leads to periods with minimum and average durations of $\min \left(T_{0}\right)=\min \left(T_{1}\right)=100 \mathrm{~ms}$ and $\mathbb{E}\left(T_{0}\right)=\mathbb{E}\left(T_{1}\right)=500$ 
ms, respectively. The resulting channel duty cycle is $50 \%$. The sequence of 600 periods is transmitted using transmitter A (see Fig. 2), which requires a total transmission time of 5 minutes.

The receiver is placed 1 metre apart from the transmitter and is configured to sense the channel (with a constant gain of $25 \mathrm{~dB}$ ) every $T_{s}$ seconds. Every $T_{s}$ seconds, the channel is sensed for $\tau=1 \mathrm{~ms}$. For the selected sample rate $\left(f_{s}=1\right.$ $\mathrm{MS} / \mathrm{s}$ ), this means that sensing decisions are made based on blocks of $N=\left\lceil\tau f_{s}\right\rceil=1000$ samples. In every sensing event, the energy of the $N$ samples is compared to a decision threshold and a binary ON/OFF decision $\left(\mathcal{H}_{0} / \mathcal{H}_{1}\right)$ is made. The sequence of binary decisions is used as shown in Fig. 4 to estimate individual period durations. At the end of the transmission/reception, the sample mean and variance of the idle periods observed at the receiver is computed and used to estimate (based on the method of moments) the parameters of the generalised Pareto distribution. This experimentally estimated distribution is then compared to the actual distribution of the transmitted periods and the accuracy of the estimation is quantified in terms of the Kolmogorov-Smirnov (KS) distance. The experiment is repeated for sensing period values from $T_{s}=10 \mathrm{~ms}$ to $T_{s}=100 \mathrm{~ms}$ (note that the condition $T_{s} \leq \mu_{i}$ is required for a correct estimation) in increments of $10 \mathrm{~ms}$ (the same sequence is transmitted for every value of $T_{s}$ ).

A key aspect of the receiver is the configuration of the energy decision threshold. In practice, the decision threshold is frequently chosen to meet a certain probability of false alarm $P_{f a}$, which only requires the receiver's noise to be known [24]. To this end, the transmitter was deactivated and the receiver was run for 5 minutes with the same configuration parameters as mentioned above and $T_{s}=10 \mathrm{~ms}$. The observed (noiseonly) energy values (30000 values in total) where employed to estimate the noise statistics of the receiver, which are shown in Fig. 5 in terms of the Cumulative Distribution Function (CDF) of the noise energy. The decision thresholds corresponding to $P_{f a}=1 \%$ (99th percentile) and $P_{f a}=10 \%$ (90th percentile) are also shown. As it can be observed in Fig. 5, the Gaussian fit corresponding to the sample mean and variance is almost exact. The KS-distance between the empiric and Gaussian fitted distributions in Fig. 5 is around 0.01 (i.e., only 1\%).

The estimation of the transmitter's distribution of idle periods was performed at the receiver for the above mentioned values of $T_{s}$ and decision thresholds corresponding to $P_{f a}=1 \%$ and $P_{f a}=10 \%$. The experimental results obtained with the developed prototype are shown in Fig. 6 along with additional results obtained with the simulation method described in [15].

The results obtained in Fig. 6 indicate that the estimation of the distribution of idle periods becomes more accurate as $T_{s}$ approaches the minimum period duration $\left(\mu_{0}\right)$ and/or $P_{f a}$ decreases. A more detailed discussion on these results, which is out of the scope of this paper, is provided in [15].

Experimental and simulation results show a good agreement for both $P_{f a}=1 \%$ and $P_{f a}=10 \%$. The main advantage of using the developed prototype is not only the shorter time required to obtain the same results but also the ability to reproduce more realistic evaluation conditions, including

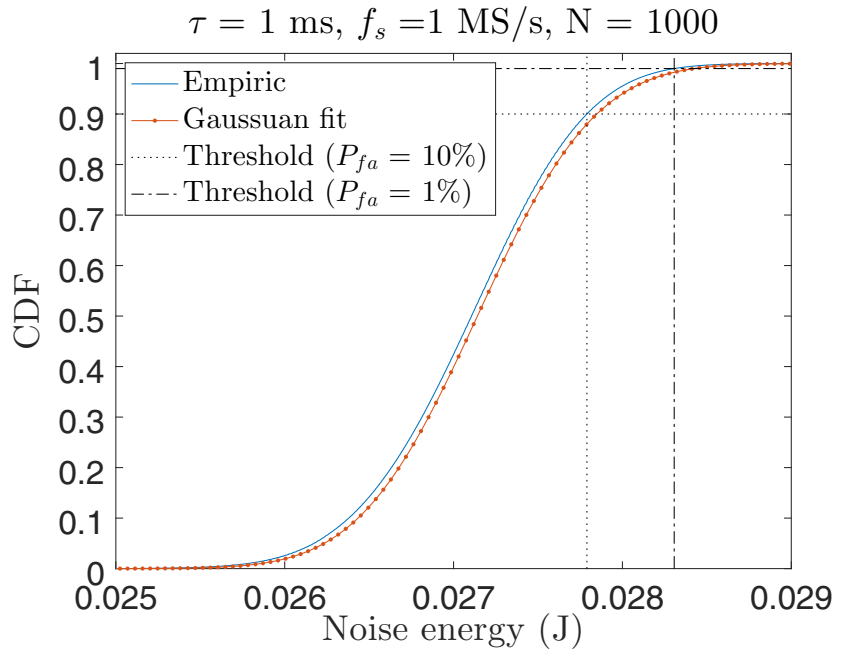

Fig. 5. Noise statistics of the receiver and selection of decision thresholds.

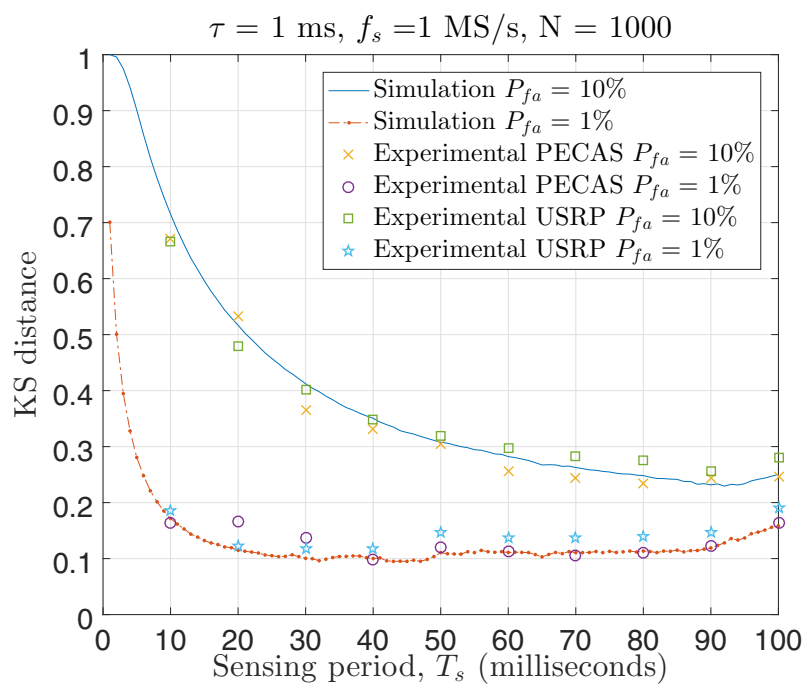

Fig. 6. Estimation error of the distribution of idle periods vs. $T_{s}$.

the channel degrading effects, practical limitations of real transmitters and receivers, and other aspects that are difficult to include (and often ignored) in simulations. Notice that the particular case study considered in this illustrative example can be carried out both with simulations and the developed prototype, however other types of studies may not be (easily) carried out by means of simulations (e.g., considering a particular radio propagation setup/environment or certain features/limitations of real hardware devices that may have a significant impact on the estimation of primary channel activity statistics).

A similar transmitter/receiver system was implemented based on two USRP B210 devices. This alternative platform is significantly (approximately 40 times) more expensive than the developed prototype. The PECAS transmitter/receiver programs were slightly modified in order to adapt them to the new hardware devices, however all transmission and reception 
procedures described in Sections III-B and IV-B were preserved. The same experiments were repeated using the same configuration (except transmitter and receiver gains of $45 \mathrm{~dB}$ and distance of half metre, needed to ensure similar signal levels). As observed in Fig. 6, there is no significant difference between the experimental results obtained with PECAS and USRP devices, which highlights the ability of the developed prototype to enable complex experiments and provide similar levels of accuracy at a much more affordable cost.

\section{CONCLUSIONS AND Discussion}

The availability of statistical information about the spectrum occupancy pattern of primary users can provide significant benefits to DSA/CR systems, which can exploit this information to predict future spectrum occupancy trends, select the most appropriate band/channel of operation, or make appropriate spectrum and radio resource management decisions to improve the DSA/CR system performance, protect primary users from interference, and increase the overall spectrum efficiency. This information can be conveniently obtained from spectrum sensing observations. However, given the practical limitations of spectrum sensing, an accurate and realistic evaluation of estimation methods based on this approach is required before the implementation in real systems.

This paper has presented the design and implementation of a low-cost Prototype for the Estimation of Channel Activity Statistics (PECAS) in DSA/CR systems. An in-depth description of the hardware design (based on common and inexpensive components) and software implementation (based on open source code freely offered to the community) has been provided, discussing implementation details that may be helpful to other researchers and engineers in the development of similar tools. While based on common and inexpensive hardware components, the developed prototype enables complex experiments, as it has been illustrated with an example, providing a level of accuracy comparable to implementations based on significantly more expensive hardware devices.

The developed prototype can be extended to embrace other experiments, for example the experimental evaluation of spectrum sensing methods for different signal formats (a wide range of low-cost amplitude, frequency and phase modulators for the $433 \mathrm{MHz}$ ISM band are available in the market) and their impact on the estimation of primary channel statistics. Moreover, the low cost of the developed prototype enables a large scale deployment of transmitters and/or receivers, thus extending the range of possible experiments (e.g., analysis of the combined effect of uncoordinated primary transmitters, or cooperative estimation of primary activity statistics).

In summary, the developed platform provides researchers and engineers with a low-cost fully functional tool for proofof-concept, validation and optimisation of algorithms and designs for the estimation of primary activity statistics.

\section{REFERENCES}

[1] Y.-C. Liang, K.-C. Chen, G. Y. Li, and P. Mähönen, "Cognitive radio networking and communications: An overview," IEEE Trans. Vehic. Tech., vol. 60, no. 7, pp. 3386-3407, Sep. 2011.
[2] M. López-Benítez, "Cognitive radio," in Heterogeneous cellular networks: Theory, simulation and deployment. Cambridge University Press, 2013, ch. 13, pp. 383-425.

[3] S. Yarkan and H. Arslan, "Binary time series approach to spectrum prediction for cognitive radio," in Proc. IEEE 66th Vehic. Tech. Conf. (VTC 2007 Fall), Sep. 2007, pp. 1563-1567.

[4] V. K. Tumuluru, P. Wang, and D. Niyato, "A neural network based spectrum prediction scheme for cognitive radio," in Proc. 2010 IEEE Int'l. Conf. Comms. (ICC 2010), May 2010, pp. 1-5.

[5] X. Liu, B. Krishnamachari, and H. Liu, "Channel selection in multichannel opportunistic spectrum access networks with perfect sensing," in Proc. 2010 IEEE Int'l. Symp. Dyn. Spect. Access Networks (DySPAN 2010), Apr. 2010, pp. 1-8.

[6] J. Vartiainen, M. Höyhtyä, J. Lehtomäki, and T. Bräysy, "Priority channel selection based on detection history database," in Proc. Fifth Int'l. Conf. Cognitive Radio Oriented Wireless Networks \& Comms. (CROWNCOM 2010), Jun. 2010, pp. 1-5.

[7] M. Höyhtyä, S. Pollin, and A. Mämmelä, "Classification-based predictive channel selection for cognitive radios," in Proc. 2010 IEEE Int'l. Conf. Comms. (ICC 2010), May 2010, pp. 1-6.

[8] F. Bouali, O. Sallent, J. Pérez-Romero, and R. Agustí, "Strengthening radio environment maps with primary-user statistical patterns for enhancing cognitive radio operation," in Proc. 6th Int'l. Conf. Cog. Radio Wireless. Netws. Comms. (CROWNCOM 2011), Jun. 2011, pp. 256-260.

[9] E. Jung and X. Liu, "Opportunistic spectrum access in multiple-primaryuser environments under the packet collision constraint," IEEE/ACM Trans. Networking, vol. 20, no. 2, pp. 501-514, Apr. 2012.

[10] S. Geirhofer, L. Tong, and B. M. Sadler, "Cognitive medium access: constraining interference based on experimental models," IEEE J. Sel. Areas Comms., vol. 26, no. 1, pp. 95-105, Jan. 2008.

[11] J. Jia, Q. Zhang, and X. Shen, "HC-MAC: A hardware-constrained cognitive MAC for efficient spectrum management," IEEE J. Sel. Areas Comms., vol. 26, no. 1, pp. 106-117, Jan. 2008.

[12] H. Su and X. Zhang, "Cross-layer-based opportunistic MAC protocols for QoS provisioning over cognitive radio wireless networks," IEEE J. Sel. Areas Comms., vol. 26, no. 1, pp. 118-129, Jan. 2008.

[13] A. Anandkumar, N. Michael, A. K. Tang, and A. Swami, "Distributed algorithms for learning and cognitive medium access with logarithmic regret," IEEE J. Sel. Areas Comms., vol. 29, no. 4, pp. 731-745, 2011.

[14] M. López-Benítez, "Sensing-based spectrum awareness in cognitive radio: challenges and open research problems," in Proc. 9th IEEE/IET Int'l. Symp. Comm. Syst. Netws. and Digital Signal Process. (CSNDSP 2014), Jul. 2014, pp. 459-464.

[15] M. López-Benítez, "Can primary activity statistics in cognitive radio be estimated under imperfect spectrum sensing?" in Proc. 24th Annual IEEE Int'l. Symp. Pers., Indoor and Mobile Radio Comms. (PIMRC 2013), Sep. 2013, pp. 750-755.

[16] W. Gabran, C.-H. Liu, P. Pawelczak, and D. Cabric, "Primary user traffic estimation for dynamic spectrum access," IEEE J. Sel. Areas Com., vol. 31, no. 3, pp. 544-558, Mar. 2013.

[17] C.-H. Liu, P. Pawelczak, and D. Cabric, "Primary user traffic classification in dynamic spectrum access networks," IEEE J. Sel. Areas Com., vol. 32, no. 11, pp. 2237-2251, Nov. 2014.

[18] J. J. Lehtomäki, M. López-Benítez, K. Umebayashi, and M. Juntti, "Improved channel occupancy rate estimation," IEEE Trans. Commun., vol. 63, no. 3, pp. 643-654, Mar. 2015.

[19] H. Urkowitz, "Energy detection of unknown deterministic signals," Proc. IEEE, vol. 55, no. 4, pp. 523-531, April 1967.

[20] T. Yücek and H. Arslan, "A survey of spectrum sensing algorithms for cognitive radio applications," IEEE Communications Surveys and Tutorials, vol. 11, no. 1, pp. 116-130, First Quarter 2009.

[21] M. López-Benítez and F. Casadevall, "Time-dimension models of spectrum usage for the analysis, design and simulation of cognitive radio networks," IEEE Trans. Vehic. Tech., vol. 62, no. 5, pp. 2091-2104, Jun. 2013.

[22] L. Devroye, Non-uniform random variate generation. Springer-Verlag, 1986.

[23] R. W. Stewart, K. W. Barlee, D. S. W. Atkinson, and L. H. Crockett, Software defined radio using MATLAB \& Simulink and the RTL-SDR. Strathclyde Academic Media, 2015.

[24] M. López-Benítez and F. Casadevall, "Improved energy detection spectrum sensing for cognitive radio," IET Communications, Special issue on Cognitive Communications, vol. 6, no. 8, pp. 785-796, May 2012. 